Research Article

\title{
Shenqi Jiangtang Granule Ameliorates Kidney Function by Inhibiting Apoptosis in a Diabetic Rat Model
}

\author{
Qian Zhang $\mathbb{D}^{D}$, Xinhua Xiao $\mathbb{D}^{\mathrm{D}}$, Jia Zheng, Ming Li, Miao Yu, Fan Ping, Tong Wang, \\ and Xiaojing Wang
}

Key Laboratory of Endocrinology, Ministry of Health, Department of Endocrinology, Peking Union Medical College Hospital, Peking Union Medical College, Chinese Academy of Medical Sciences, Beijing 100730, China

Correspondence should be addressed to Xinhua Xiao; xiaoxh2014@vip.163.com

Received 10 September 2019; Accepted 30 October 2019; Published 20 November 2019

Academic Editor: Ronald Sherman

Copyright (c) 2019 Qian Zhang et al. This is an open access article distributed under the Creative Commons Attribution License, which permits unrestricted use, distribution, and reproduction in any medium, provided the original work is properly cited.

Diabetic nephropathy (DN) is a major microvascular complication of diabetes. In addition to moderating hyperglycemia, Shenqi Jiangtang Granule (SJG) had a beneficial effect on kidney function in a clinical trial. However, the mechanism involved remains unclear. This study was conducted to identify the underlying molecular mechanisms. A diabetic rat model was generated by using a high-fat diet and streptozotocin (STZ) injection. Then, rats were given SJG at dosages of $400 \mathrm{mg} / \mathrm{kg} / \mathrm{d}$ or $800 \mathrm{mg} / \mathrm{kg} / \mathrm{d} \mathrm{by} \mathrm{gavage}$ for 8 weeks. After 8 weeks of treatment, blood glucose, serum creatinine, blood urea nitrogen (BUN), and 24-h urinary albumin were measured. Histochemical staining and TdT-mediated dUTP nick-end labeling (TUNEL) assays were performed in kidney. Kidney genomic expression in the SJG-treated group and diabetic group was detected by using a genome expression microarray. We found that SJG treatment reduced blood glucose, serum creatinine, BUN, and 24-h urinary albumin and affected kidney histology. The gene array revealed that the expression of 99 genes increased and the expression of 91 genes decreased in the HSJG group, compared with those of in the diabetic group. Pathway and gene ontology analysis of the differentially expressed genes showed an enrichment of the apoptosis pathway. SJG treatment reduced TUNEL- and caspase-3-positive cells in diabetic kidneys. SJG upregulated Bcl-2 and regucalcin expressions and reduced casp 3 and Apaf1 expressions in diabetic rats. Our results suggest that SJG exerts a renal protective effect through the inhibition of cell apoptosis in a diabetic rodent model.

\section{Introduction}

The number of patients with diabetes is dramatically increasing worldwide [1]. Forty percent of diabetic patients develop diabetic nephropathy (DN), which is a major microvascular complication [2]. In addition, $\mathrm{DN}$ is the most common cause of end-stage renal disease [3]. DN is also a major cause of morbidity and mortality in patients with kidney disease worldwide.

The pathological character of DN includes abnormal accumulation of extracellular matrix (ECM), thickening and hypertrophy of the glomerular basement membrane, and loss of glomerular and tubular cells, which leads to kidney fibrosis [4]. These structural changes cause increased proteinuria and albumin excretion and reduced glomerular filtration rate (GFR).
In addition to hyperglycemia, numerous pathways contribute to the development of $\mathrm{DN}$, including oxidative stress [5], proinflammatory molecules [6], enhanced reactive oxygen species (ROS) [7], activation of protein kinase C [8], increased formation of advanced glycation end products (AGEs) [9], and the renin-angiotensin system (RAS).

Shenqi Jiangtang Granule (SJG) comprises Panax ginseng (Panax ginseng C.A. Mey), Radix astragali (Astragalus penduliflorus Lam.), Radix rehmannia (Rehmannia glutinosa (Gaertn.) DC.), Radix trichosanthis (Trichosanthes kirilowii Maxim), Fructus Schisandrae chinensis (Schisandra chinensis (Turcz.) Baill), Ophiopogonis radix (Ophiopogon japonicus (Thunb.) Ker Gawl), Fructus lycii (Lycium chinense Mill), Fructus rubi (Rubus chingii $\mathrm{Hu}$ ), Rhizoma dioscoreae (Dioscorea oppositifolia L.), Alismatis rhizome (Alisma plantago-aquatica subsp.), and Poris cocos (Smilax glabra 
Roxb). SJG is approved by the State Food and Drug Administration of China (State medical license no. Z10950075). A clinical trial indicates that single drug treatment with SJG has an antidiabetic effect in type 2 diabetic patients, compared with that of exercise or dietary intervention [10]. A meta-analysis showed that SJG combination therapy has a better effect than traditional therapy alone, including reducing urinary albumin excretion rate (UAER), serum blood urea nitrogen (BUN), serum creatinine, and $24 \mathrm{~h}$ urea albumin in diabetic patients [11]. The mechanism driving this may be involved in inducing HIF- $1 \alpha$ and HO-1 expression [12]. Recently, in vitro experiment revealed SJG as an alphaglucosidase inhibitor [13]. However, the exact mechanism underlying the beneficial effect of SJG on kidney function is still unknown.

We hypothesized that SJG has multiple targets in the kidney, thus moderating the kidney fibrosis in diabetic rats. Traditional Chinese herbs usually have multiple targets. Therefore, we used a genome-wide array strategy in kidney and pathway analysis.

\section{Methods}

2.1. Medicine. Shenqi Jiangtang Granule includes Panax ginseng (Panax ginseng C.A. Mey, 0.71\%), Radix astragali (Astragalus penduliflorus Lam, 14.71\%), Radix rehmannia (Rehmannia glutinosa (Gaertn.) DC., 22.06\%), Radix trichosanthis (Trichosanthes kirilowii Maxim, 7.35\%), Fructus Schisandrae chinensis (Schisandra chinensis (Turcz.) Bail, 7.35\%), Ophiopogonis radix (Ophiopogon japonicus (Thunb.) Ker Gawl, 7.35\%), Fructus lycii (Lycium chinense Mill, 14.71\%), Fructus rubi (Rubus chingii Hu, 3.7\%), Rhizoma dioscoreae (Dioscorea oppositifolia L., 7.35\%), Alismatis rhizome (Alisma plantago-aquatica subsp., 7.35\%), and Poris cocos (Smilax glabra Roxb., 7.35\%). SJG was provided by Lunan Hope Pharmaceutical Co., Ltd. (Shandong, China). The eleven component herbs, Panax ginseng (6 g), Radix astragali (124 g), Radix rehmannia (186 g), Radix trichosanthis (62 g), Fructus Schisandrae chinensis (62 g), Ophiopogonis radix (62 g), Fructus lycii (124 g), Fructus rubi (31 g), Rhizoma dioscoreae (62 g), Alismatis rhizome (62 g), and Poris cocos (62 g), were soaked in 60\% ethanol for $1 \mathrm{~h}$ and extracted twice by refluxing for $2 \mathrm{~h}$. The condensed extracts were mixed with dextrin and sugar powder to make up SJG.

2.2. High-Performance Liquid Chromatography (HPLC) Analysis of SJG. SJG powder $(4.0 \mathrm{~g})$ was refluxed 4 times by $n$-butyl alcohol. Then, the combined extracts were concentrated by vacuum-rotary evaporation. The concentration was eluted by $70 \%$ ethanol on macroporous adsorptive resin. All the collected eluents were concentrated and then dried at $40^{\circ} \mathrm{C}$ in a vacuum oven. Final residue of SJG was dissolved in ethanol and then filtered through a $0.45 \mu \mathrm{m}$ filter membrane. SJG was characterized by using an Agilent 1260 Infinity II HPLC (Agilent Technologies, CA, USA) with a Symmetry C18 column $(150 \mathrm{~mm} \times 4.6 \mathrm{~mm}$, i.e., particle size $5 \mu \mathrm{m}, \mathrm{MA}$, USA). The column was eluted at $30^{\circ} \mathrm{C}$ with a detection wavelength at $203 \mathrm{~nm}$ and an injection volume of $10 \mu \mathrm{L}$. The flow rate of the mobile phase of acetonitrile (A) and water (B) was set at $1.0 \mathrm{~mL} / \mathrm{min}$. Gradient separation was based on the following: $0-12 \mathrm{~min}, 0.5-5 \% \mathrm{~A} ; 12-38 \mathrm{~min}, 5-23 \% \mathrm{~A}$; 38-78 min, 23-40\% A; 78-99 min, 40-61\% A; 99-108 min, 61-80\% A; and 108-120 $\min , 80 \% \mathrm{~A}$.

2.3. Animal Treatments. Twenty-four male Sprague Dawley rats were purchased from the Institute of Laboratory Animal Sciences of the Chinese Academy of Medical Sciences and Peking Union Medical College in Beijing, China (SCXK2014-0013) weighing 170 to $190 \mathrm{~g}$ each. They were placed in a 12-h light/dark cycle and $25^{\circ} \mathrm{C}$ controlled housing and were given food and water ad libitum. A diabetic model was generated by an intraperitoneal injection of streptozotocin (60 mg/kg, STZ, Sigma, St. Louis, MO). A week after the injection, the diabetes model was confirmed. Rats with fasting blood glucose levels greater than $11.1 \mathrm{mmol} / \mathrm{L}$ were considered diabetic. Diabetic rats were randomly divided into three groups ( $n=6$ per group): a low dose of Shenqi Jiangtang Granule (LSJG) group, a high dose of Shenqi Jiangtang Granule (HSJG) group, and a diabetic group. The typical human daily dose of Shenqi Jiangtang Granule (SJG) is $3 \mathrm{~g}$ per $50 \mathrm{~kg}$ of body weight. LSJG and HSJG were administered oral Shenqi Jiangtang Granule at $400 \mathrm{mg} / \mathrm{kg} /$ day or $800 \mathrm{mg} / \mathrm{kg} /$ day for 8 weeks. The dosage of LSJG and HSJG for rats is corresponded to $3 \mathrm{~g} /$ day and $6 \mathrm{~g}$ /day for humans.

The diabetic and control groups received an equal volume of saline. After 8 weeks of treatment, all rats were anesthetized with intraperitoneal injection of sodium pentobarbital $(150 \mathrm{mg} / \mathrm{kg})$ and then sacrificed. Kidneys were immediately removed. All animal treatment and procedures were approved by the Animal Care Committee of the Peking Union Medical Hospital Animal Ethics Committee (Project XHDW-2015-0051, 15 February 2015), and all efforts were made to minimize suffering.

2.4. Metabolic Parameter Analysis. After $6 \mathrm{~h}$ of fasting, blood was collected. Serum was isolated from the blood samples by centrifuging at $3000 \mathrm{~g}$ for $10 \mathrm{~min}$ (Heraeus Varifuge 3.0, Hamburg, Germany). Serum creatinine and blood urea nitrogen (BUN) were measured with a Beckman Coulter AU5800 analyzer (Beckman, Germany). At the end of the 8week treatment, 24-h urine samples were collected using metabolic cages, centrifuged at $3000 \mathrm{~g}$ for $10 \mathrm{~min}$ (Heraeus Varifuge 3.0, Hamburg, Germany) and stored at $-80^{\circ} \mathrm{C}$. Urine albumin concentration was measured by a Beckman Counter AU5800 analyzer (Beckman, Germany).

2.5. Histology. Kidneys were fixed with $4 \%$ formalin and embedded in paraffin. Kidney sections $(5 \mu \mathrm{m})$ were stained with periodic acid Schiff (PAS). The glomerular damage score of each rat was calculated as the arithmetic mean of 60 glomeruli ( $\times 400$ magnification) [14]. Briefly, glomerular lesions were graded as normal (or minimal) to severe (extensive damage). Severity was graded as absent/normal (grade 0), mild (grade 1), moderate (grade 2), and severe 
TABLE 1: Oligonucleotide sequences for qPCR analysis.

\begin{tabular}{lcccc}
\hline Gene symbol & Gene bank ID & Forward primer & Reverse primer & Product size (bp) \\
\hline Rgn & NM_031546 & GGGTGGCCTGTTACAATGGA & ATCCTTCCCTCCAAAGCAGC & 119 \\
Apaf1 & NM_023979 & GTAGACGGCTTTCTCCGCTC & CGGATCCAGGACACAAAAGC & 203 \\
Bcl2 & NM_016993 & GAGGGGCTACGAGTGGGATA & CGGTAGCGACGAGAGAAGTC & 243 \\
Casp3 & NM_012922 & GAGCTTGGAACGCGAAGAAA & CCATTGCGAGCTGACATTCC & 224 \\
\hline
\end{tabular}

Rgn: regucalcin; Apaf1: apoptotic peptidase activating factor 1; Casp3: caspase 3.

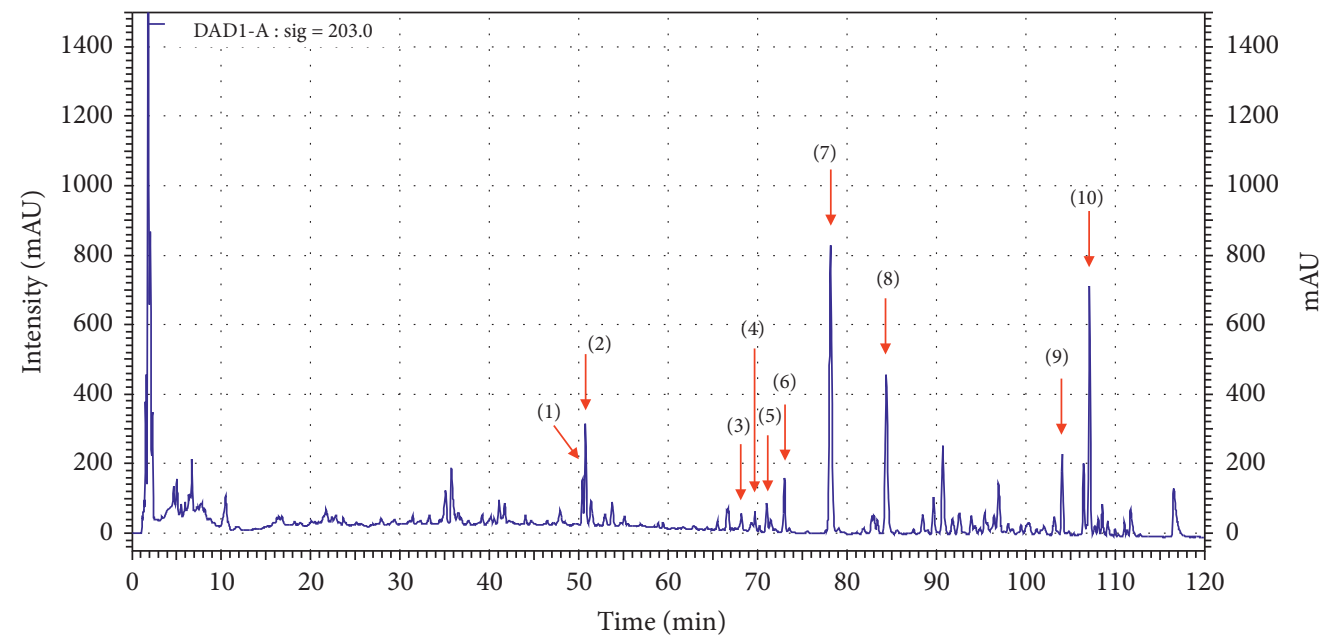

Figure 1: HPLC analysis of SJG. (1) Rg1 (ginsenoside Rg1), (2) Re (ginsenoside Re), (3) Rb1 (ginsenoside Rb1), (4) Rc (ginsenoside Rc), (5) astragaloside IV, (6) Rd (ginsenoside Rd), (7) schisandrin, (8) schisandrol B, (9) deoxyschisandrin, and (10) $\gamma$-schisandrin.

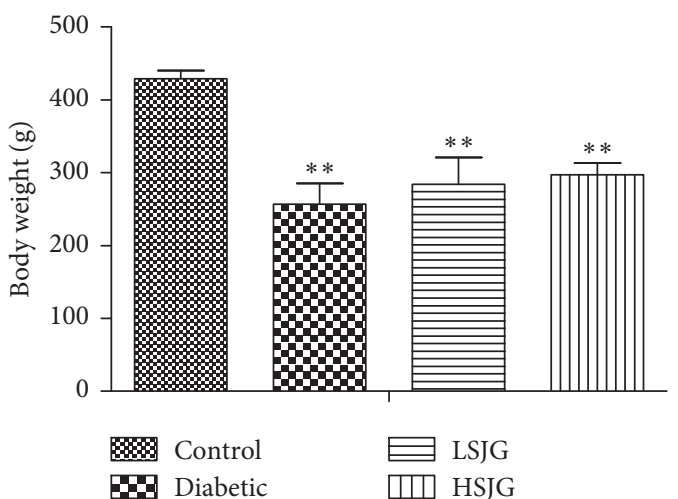

(a)

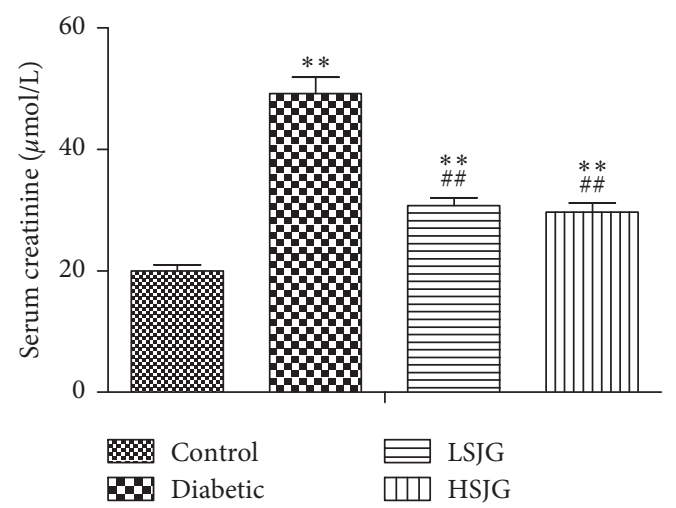

(c)

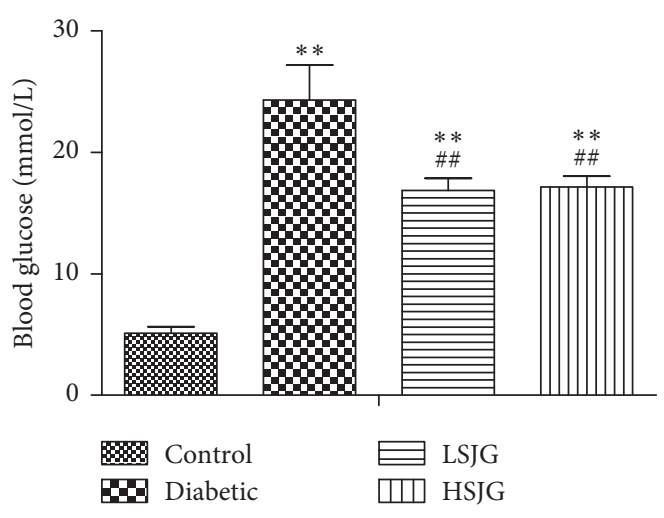

(b)

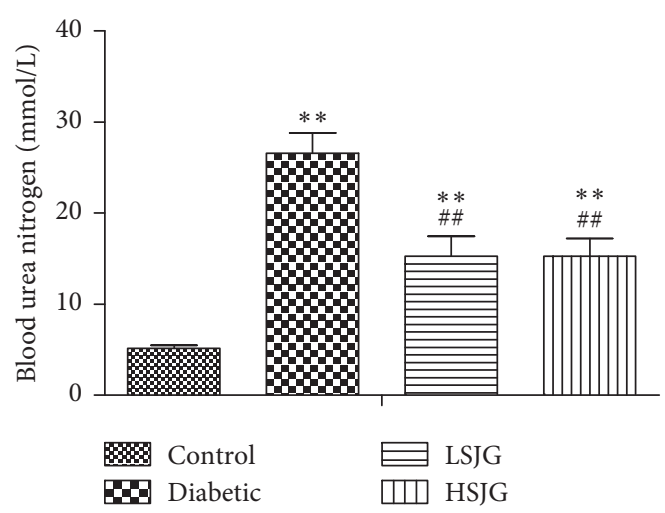

(d)

Figure 2: Continued. 


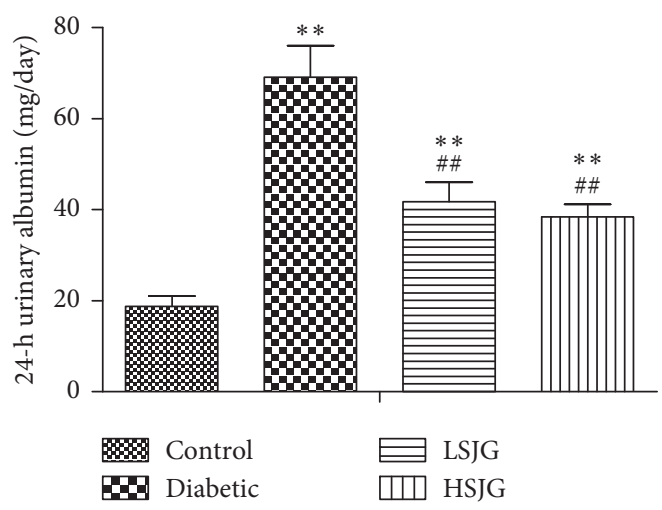

(e)

FIgURE 2: The effect of SJG on body weight (a), blood glucose (b), serum creatinine (c), blood urea nitrogen (d), and 24-h urinary albumin (e). Values are mean \pm S.D. $(n=6),{ }^{* *} P<0.01$ compared with the control group, and ${ }^{\# \#} P<0.01$ compared with the diabetic group. SJG: Shenqi Jiangtang Granule.

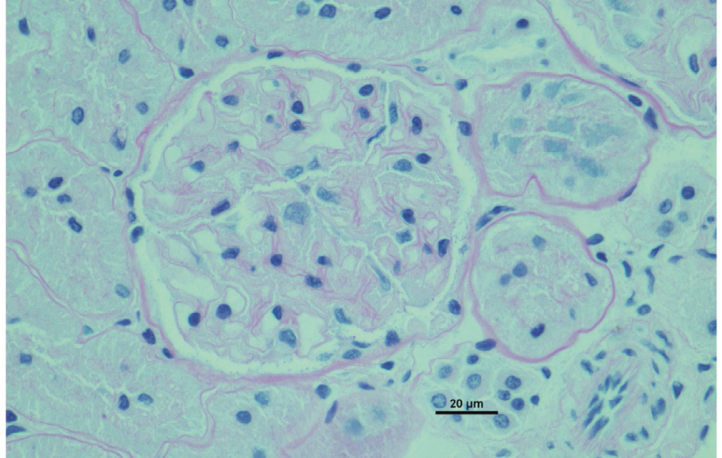

Control

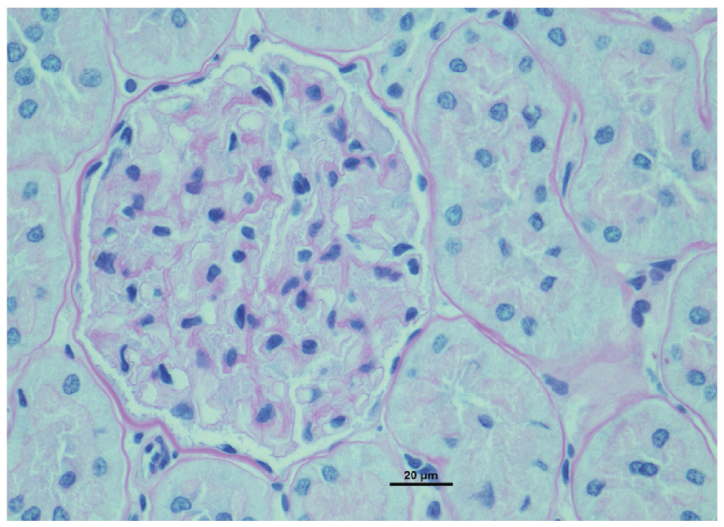

LSJG

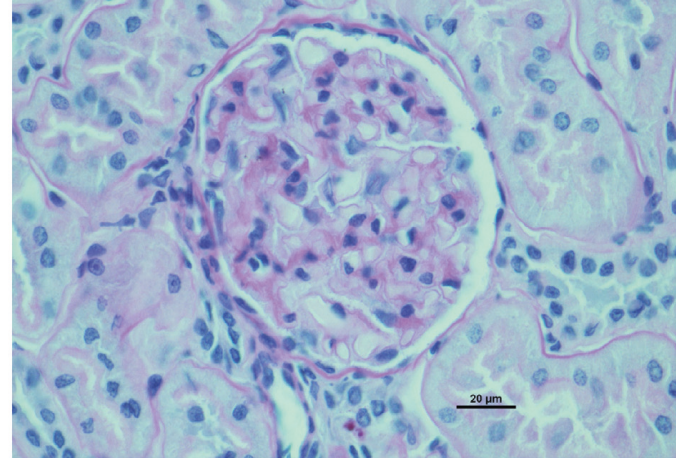

Diabetic

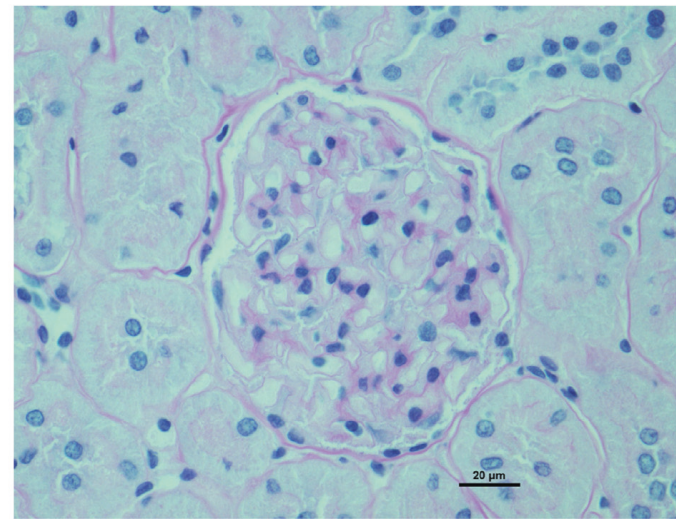

HSJG

(a)

Figure 3: Continued. 


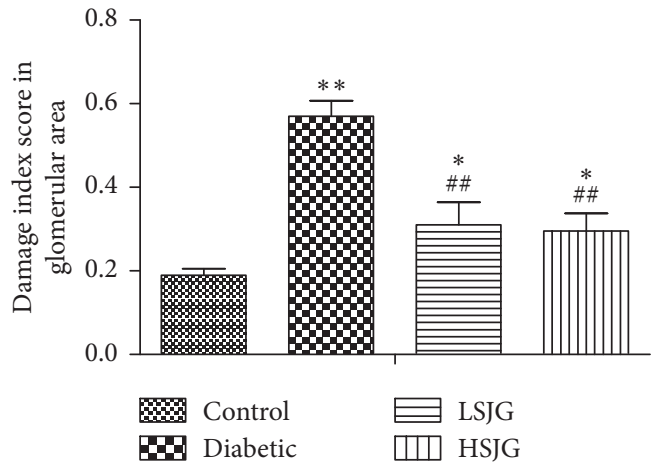

(b)

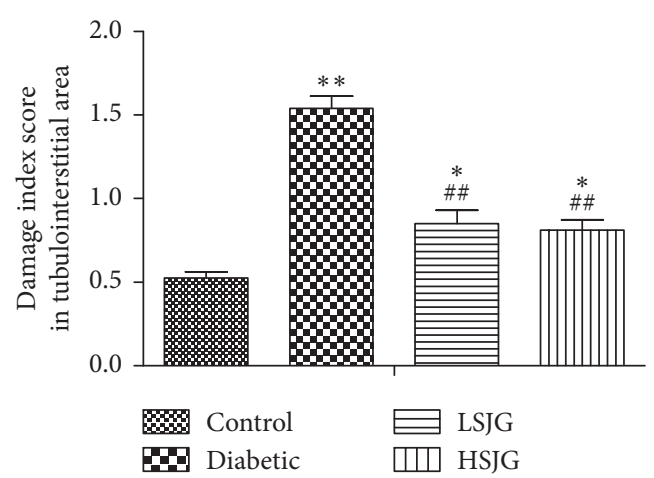

(c)

FIGURE 3: The effect of SJG on kidney histology. PAS stained kidneys (400x magnification) (a) and glomerular (b) and tubulointerstitial (c) damage index scores in kidney. Values are mean \pm S.D. $(n=6),{ }^{*} P<0.05$, and ${ }^{* *} P<0.01$ compared with the normal group; ${ }^{\# \#} P<0.01$ compared with the diabetic group. SJG: Shenqi Jiangtang Granule.

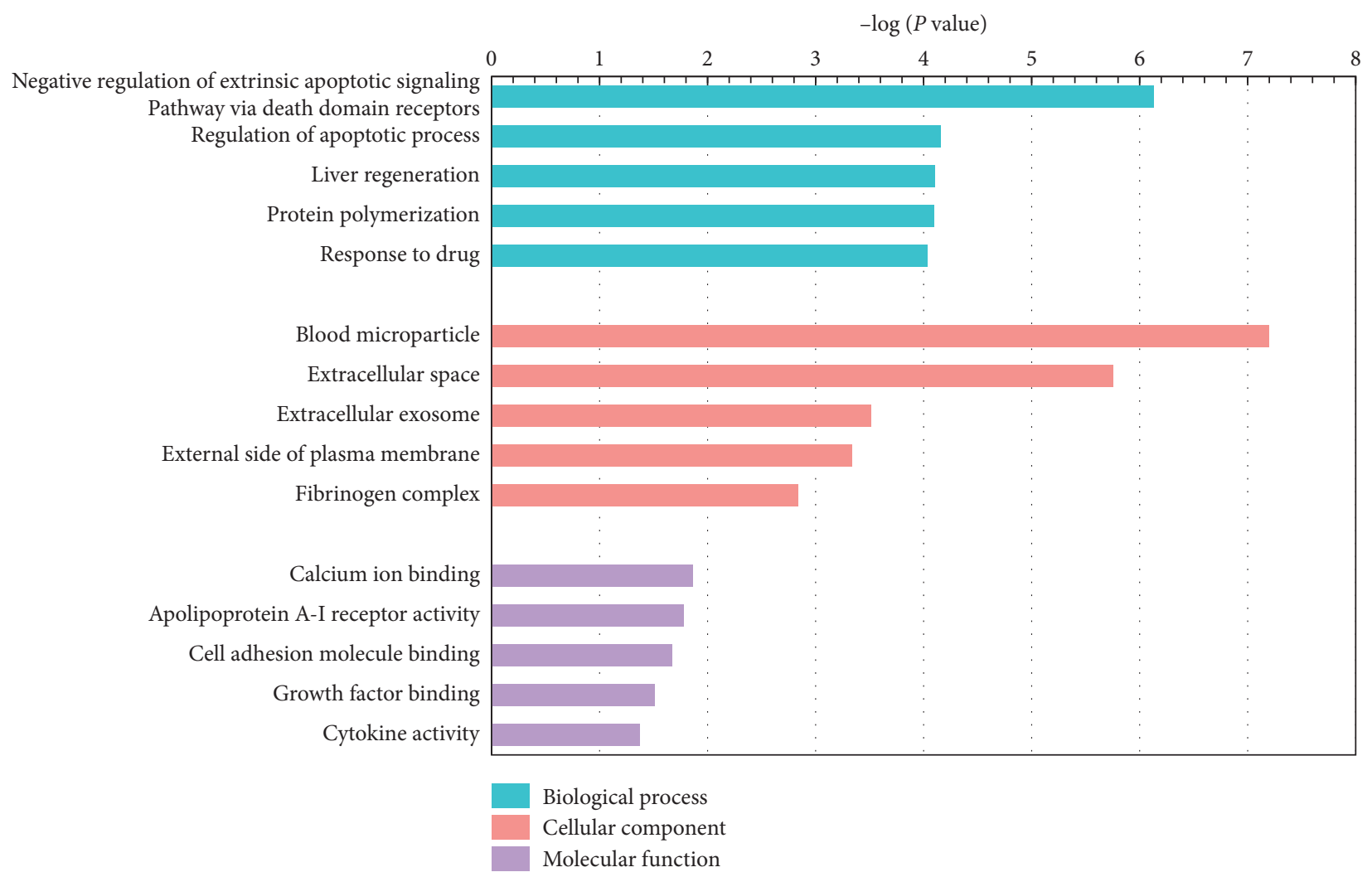

FIgURE 4: The enriched GO terms with differentially expressed genes. Top five terms in biological process (BP), cellular component (CC), and molecular function (MP).

(grade 3). The tubular damage (dilation, atrophy, hyaline in tubular lumen, infiltration of mononuclear cells, and interstitial fibrosis) was assessed as previously described with a semiquantitative method from Grade 0 (normal) to Grade 3 (severe) [15]. Tubulointerstitial damage scores are given as the arithmetic mean of all fields ( $\times 400$ magnification).

2.6. RNA Extraction and Gene Expression Array. Total RNA was extracted from the rat kidney cortex using a mirVana ${ }^{\mathrm{TM}}$ RNA Isolation Kit (Ambion, San Paulo, Brazil). Double- stranded cDNA was synthesized from total RNA. Then, cDNA was labeled with biotin. The biotinylated cDNA was purified, fragmented, and hybridized to an Affymetrix GeneChip Rat Gene 2.0 ST whole transcript-based array (Affymetrix Technologies, Santa Clara, CA). Genes were selected based on $P<0.05$ and fold change $>1.5$. The data obtained have been deposited in the NCBI Gene Expression Omnibus (GEO) database (accession number GSE134071.

DAVID (Database for Annotation, Visualization, and Integrated Discovery) web-based software tool was used to 


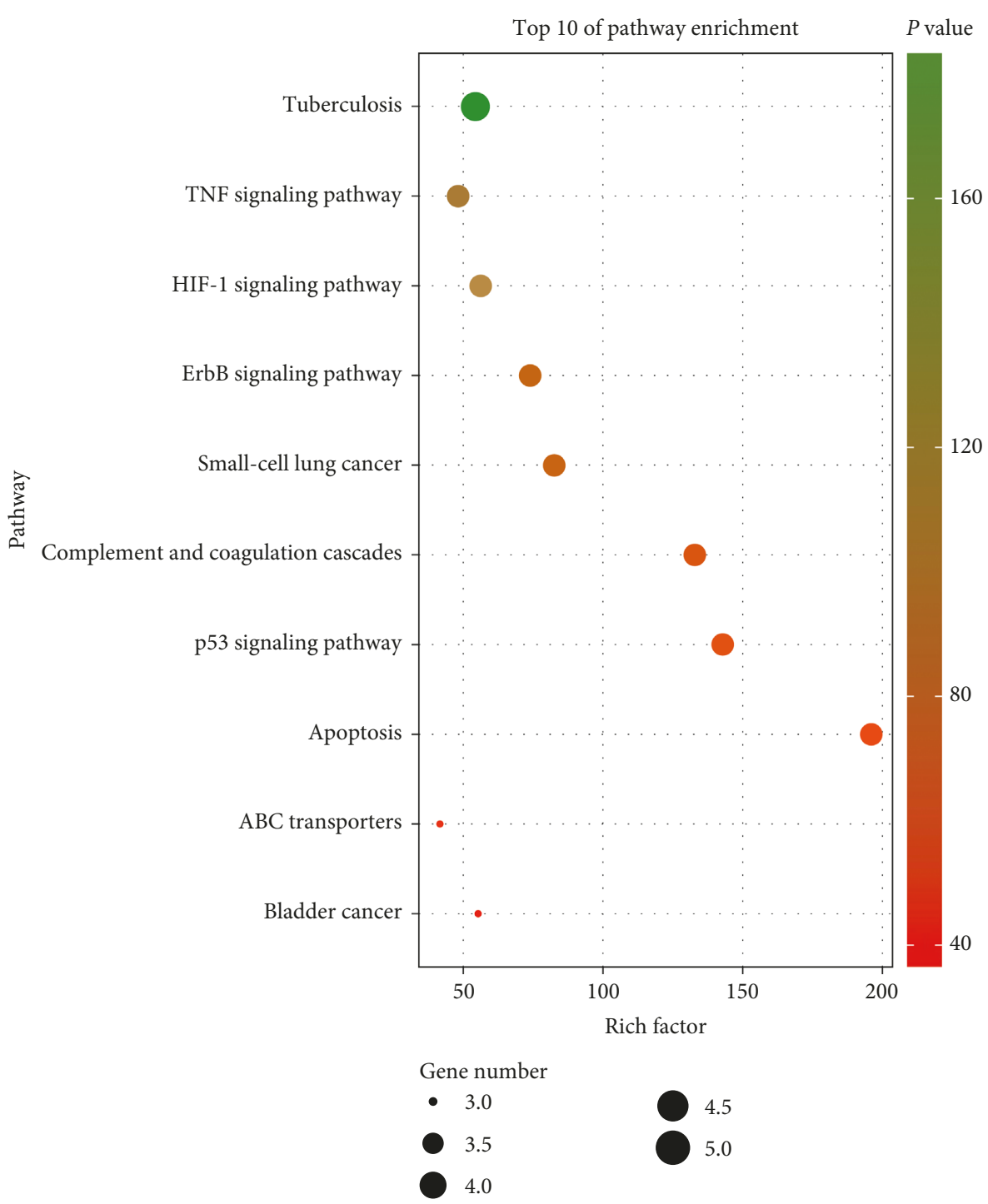

Figure 5: Top ten KEGG pathways enrichment point diagram in the SJG group compared with the diabetic group. The vertical axis represents the pathway name, the horizontal axis represents the rich factor, the size of the dot indicates the number of genes expressed in the pathway, and the color of the dot corresponds to the different $P$ values.

perform gene ontology (GO) enrichment analysis. The Kyoto Encyclopedia of Genes and Genomes (KEGG) database was utilized to identify pathways. For interaction network analysis, the STRING (Search Tool for the Retrieval of Interacting Genes) database was used.

2.7. Real-Time PCR. Total RNA from the kidney cortex was used to synthesize cDNA using SuperScript II reverse transcriptase (Life Technologies, Carlsbad, CA). Quantitative PCR was carried out by using specific primers (Table 1). A SYBR Green Mix Kit (Applied Biosystems, Foster City, CA) and an ABI Prism 7500 Real-Time System (Applied Biosystems, Foster City, CA) were used for PCR. Relative expression levels were calculated with the $2^{-\Delta \Delta \mathrm{Ct}}$ method.

2.8. TUNEL Assay. Apoptosis was detected by a terminal deoxynucleotidyl transferase-mediated dUTP nick-end labeling (TUNEL) assay, using an Apoptosis Detection Kit (Roche Applied Science, Mannheim, Germany). Briefly, paraffin-embedded kidney sections were dewaxed, washed with PBS, and incubated with Proteinase K for $20 \mathrm{~min}$. After washing with PBS, sections were incubated with the TUNEL reaction mix at $37^{\circ} \mathrm{C}$ for $60 \mathrm{~min}$.

2.9. Immunohistochemistry for Bcl-2 and Caspase-3 in Kidney. The kidneys were fixed in $4 \%$ paraformaldehyde and embedded in paraffin. Then, $5 \mu \mathrm{m}$-thick sections were dewaxed. After washing in PBS, sections were incubated with $1.5 \%$ $\mathrm{H}_{2} \mathrm{O}_{2}$ in methanol to block endogenous peroxidase activity. Nonspecific binding was blocked with $10 \%$ normal goat serum in PBS. Sections were incubated overnight with, and rabbit polyclonal caspase-3 (1:50, Santa Cruz Biotechnology, Dallas, TX) at $4^{\circ} \mathrm{C}$. Then, the sections were washed with PBS and incubated with a horseradish peroxidase- (HRP-) 


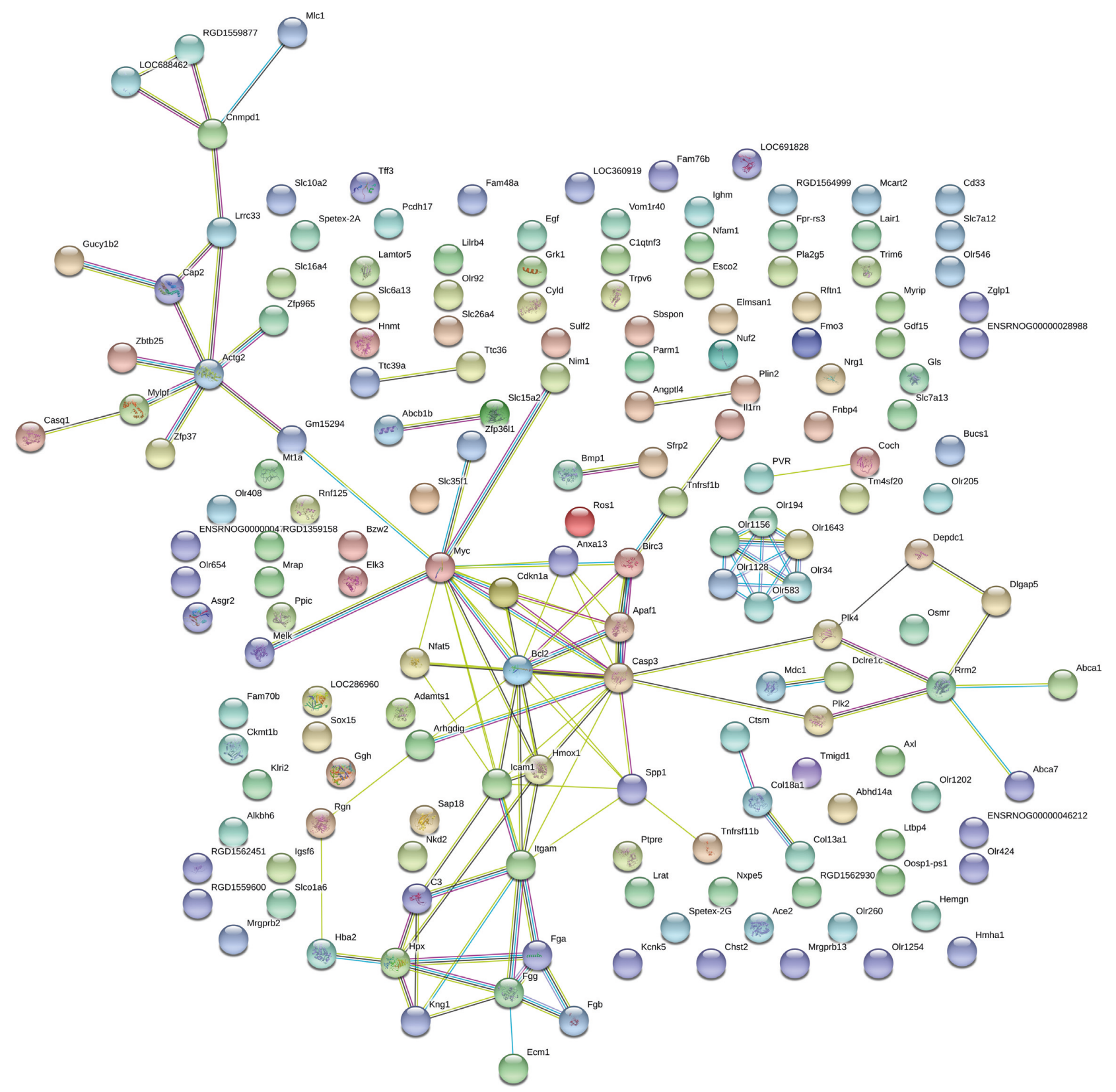

FIgURe 6: Protein-protein interaction network in the SJG group compared with the diabetic group. The nodes stand for differentially expressed genes in the SJG group compared with the diabetic group. The lines stand for the interactions between two proteins.

conjugated secondary antibody $(1: 2000$, Santa Cruz Biotechnology, Dallas, TX) for $1 \mathrm{~h}$ at room temperature. After washing with PBS, the sections were stained with a $3,3^{\prime}$ diaminobenzidine (DAB) Color Development Kit (ZSGBBIO, Beijing, China). The slides were analyzed using ImageJ software (National Institutes of Health, Baltimore, MD) at 400x magnification. Five slides were analyzed, and six rats were in each group.

2.10. Statistical Analysis. Data are shown as mean $\pm \mathrm{SD}$. Statistical analyses were conducted with two-way ANOVA followed by Tukey's post hoc test. Graphpad Prism 6
(GraphPad Software Inc., CA, USA) was used for data analysis. $P<0.05$ was set for significance definition.

\section{Results}

3.1. HPLC Analysis of SJG. Ten main components of SJG were confirmed by HPLC analysis. The UV detector for HPLC analysis was set to $203 \mathrm{~nm}$ according to the standard maximum absorption rate. The HPLC analysis of SJG is presented in Figure 1. Ten main constituents of SJG are (1) ginsenoside Rg1 (15.95 mg/g), (2) ginsenoside Re (27.63 mg/ g), (3) ginsenoside Rb1 (2.21 mg/g), (4) ginsenoside Rc (4.47 mg/g), (5) astragaloside IV (0.31 mg/g), (6) ginsenoside 


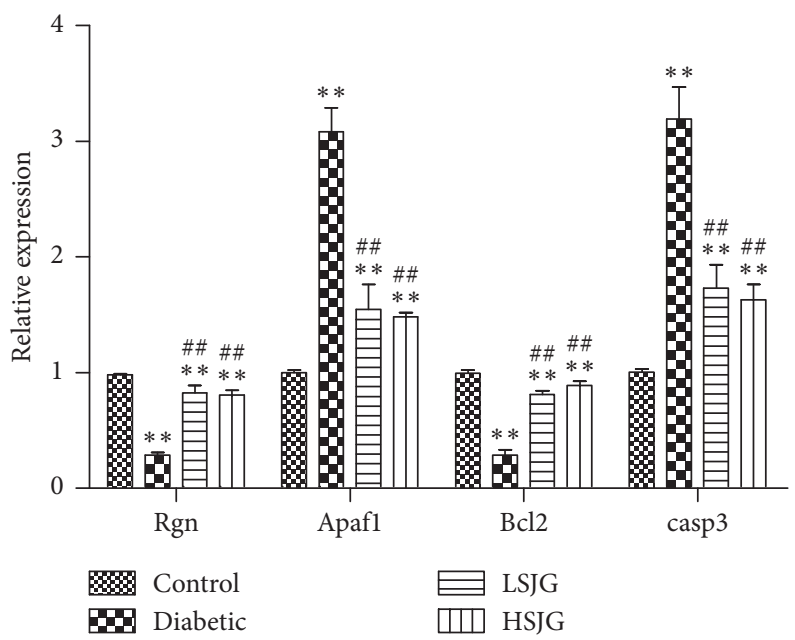

Figure 7: Confirmation of four representative differentially expressed genes by qPCR. Values are mean \pm S.D. $(n=6),{ }^{* *} P<0.01$ compared with the normal group, and ${ }^{\# \#} P<0.01$ compared with the DN group. Rgn: regucalcin; Apaf1: apoptotic peptidase activating factor 1; and Casp3: caspase 3.

Rd (14.65 mg/g), (7) schisandrin (2.96 mg/g), (8) schisandrol B (0.75 mg/g), (9) deoxyschisandrin $(0.46 \mathrm{mg} / \mathrm{g})$, and (10) $\gamma$-schisandrin $(1.29 \mathrm{mg} / \mathrm{g})$.

\subsection{SJG Showed No Influence on Body Weight in Diabetic} Rats. As shown in Figure 2(a), the mean body weight of diabetic rats decreased significantly compared with that of the control rats $(P<0.01)$.

3.3. SJG Reduced FBG in Diabetic Rats. The diabetic rats had significantly higher FBG than the control rats $(P<0.01$, Figure 2(b)). SJG significantly reduced the FBG in diabetic rats $(P<0.01$, Figure $2(b))$ compared with the diabetic rats.

3.4. SJG Moderated Kidney Dysfunction in Diabetic Rats. The serum creatinine, blood urea nitrogen, and 24-h urinary albumin increased in the diabetic group compared with the control group $(P<0.01$, Figures $2(\mathrm{c})-2(\mathrm{e}))$. SJG significantly reduced serum creatinine, blood urea nitrogen, and $24-\mathrm{h}$ urinary albumin $(P<0.01$, Figures $2(\mathrm{c})-2(\mathrm{e}))$ compared with the diabetic rats.

3.5. SJG Affected Renal Histology in Diabetic Rats. Compared with the control group, the kidneys of diabetic rats showed increased glomerular hypertrophy and tubulointerstitial changes (Figures 3(a)-3(c)). SJG treatment significantly reduced both the glomerular and tubulointerstitial changes (Figures 3(a)-3(c)) compared with the diabetic rats.

3.6. Gene Array Results, GO, Pathway, and Network Analysis. Ninety-nine genes increased and 91 genes decreased in the HSJG group, compared with the diabetic group (fold change $>1.5, P<0.05)$. To identify the relevant pathways and perform gene ontology, we analyzed the differentially expressed genes in the HSJG group vs. the control group using the DAVID. We identified significant regulation of five biological processes (BPs), cellular components (CCs), and molecular functions (MFs), respectively. Negative regulation of the extrinsic apoptotic signaling pathway via death domain receptors (involved gene number $=6$ ), regulation of apoptotic process (involved gene number $=9$ ), liver regeneration (involved gene number $=6$ ), protein polymerization (involved gene number $=4$ ), and response to drug (involved gene number $=15)$ were the top five terms of the BP section (Figure 4). In pathway analysis, we identified significant regulation of ten signaling pathways, including apoptosis (involved gene number $=4$ ), p53 signaling pathway (involved gene number $=4)$, complement and coagulation cascades (involved gene number $=4$ ), small-cell lung cancer (involved gene number $=4$ ), ErbB signaling pathway (involved gene number $=4$ ), bladder cancer (involved gene number $=3$ ), HIF-1 signaling pathway (involved gene number =4), ABC signaling pathway (involved gene number $=3$ ), TNF signaling pathway (involved gene number $=4$ ), and tuberculosis (involved gene number $=5$, Figure 5).

The 190 differentially expressed genes were mapped using the String online software. We found that 171 nodes (genes) had 114 joint edges (interactions) among these genes (Figure 6). In these nodes and edges, 11 nodes (each node with more than 5 joint edges) had 97 joint edges, representing all of the nodes. These 11 nodes are myelocytomatosis oncogene (Myc), caspase-3 (Casp3), Bcl2, integrin, alpha M (Itgam), intercellular adhesion molecule 1 (Icam1), actin, gamma 2, smooth muscle, enteric (Actg2), heme oxygenase 1 (Hmox1), apoptotic peptidase activating factor 1 (Apaf1), fibrinogen gamma chain (Fgg), hemopexin ( $\mathrm{Hpx})$, and secreted phosphoprotein 1 (Spp1). These genes may have important functions in kidney function affected by SJG treatment.

3.7. Real-Time PCR. Four differentially expressed genes were analyzed using real-time PCR. We found that regucalcin 

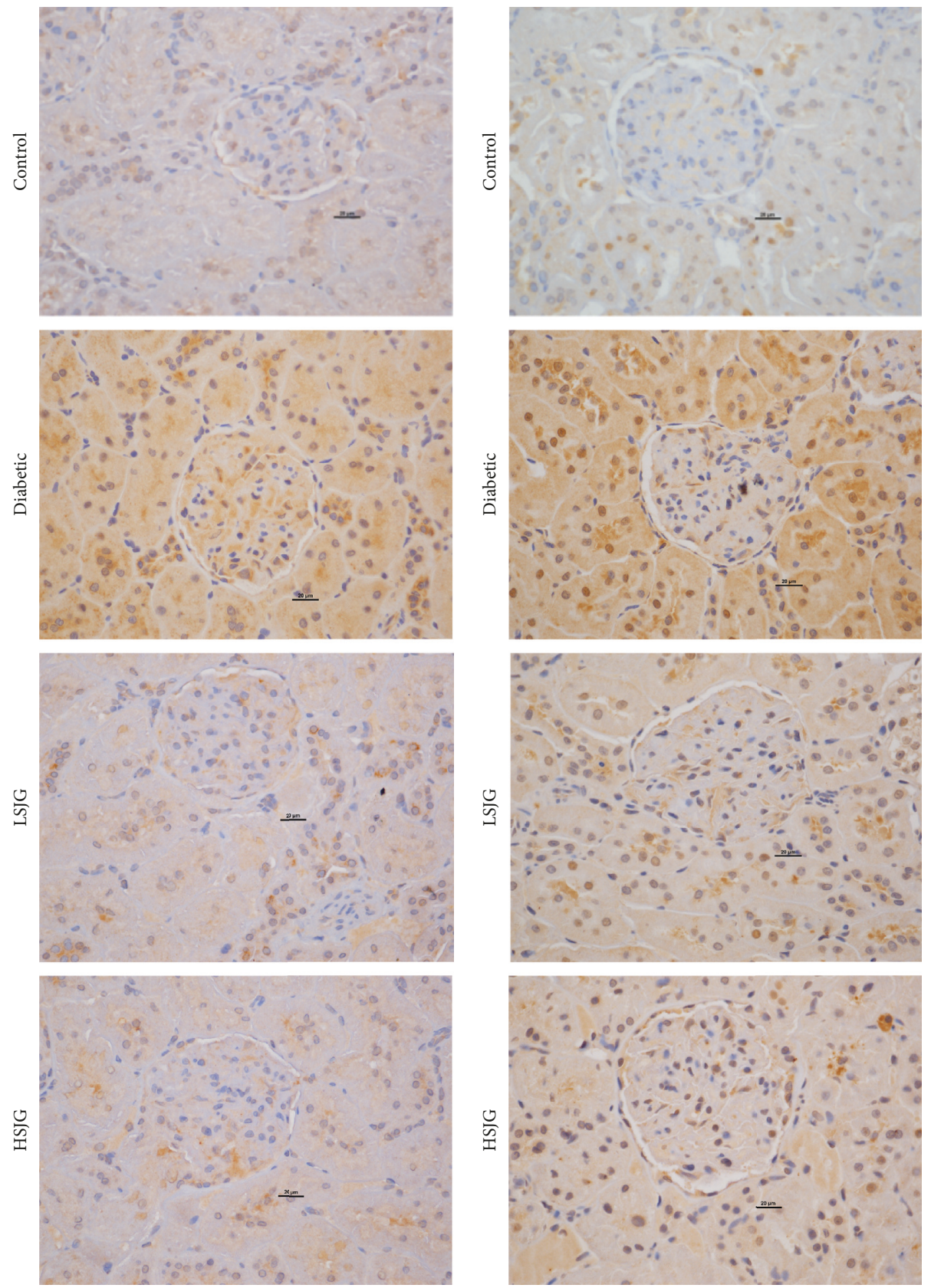

(a)

(b)
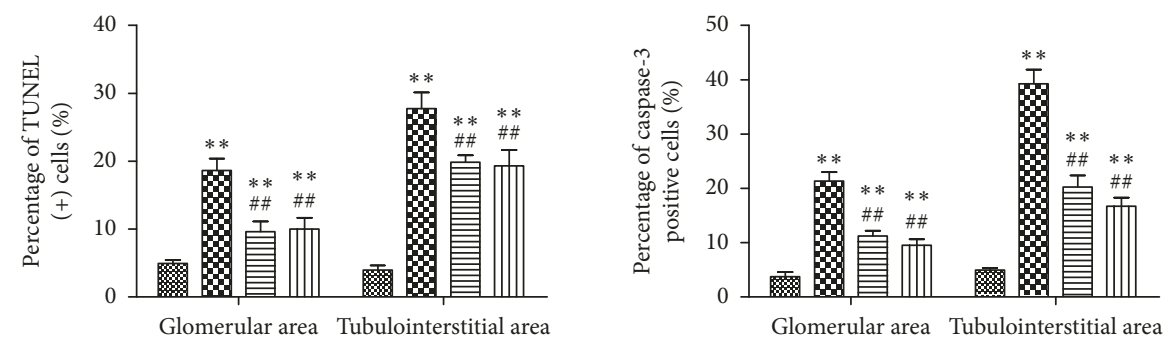

Glomerular area Tubulointerstitial area

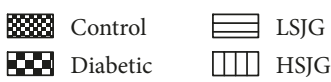

$\begin{array}{ll}\text { 80 Control } & \equiv \text { LSJG } \\ \text { Diabetic } & \text { एाI HSJ }\end{array}$

(c)

(d)

Figure 8: The effect of SJG on TUNEL (+) cells and caspase-3 expression. (a) TUNEL assay, (b) immunostaining for caspase-3, (c) percentage $(\%)$ of TUNEL $(+)$ cells in kidney, and $(d)$ percentage $(\%)$ of caspase-3 $(+)$ cells in kidney. Values are mean \pm S.D. $(n=6)$, ${ }^{* *} P<0.01$ compared with the normal group, and ${ }^{\# \#} P<0.01$ compared with the diabetic group. 


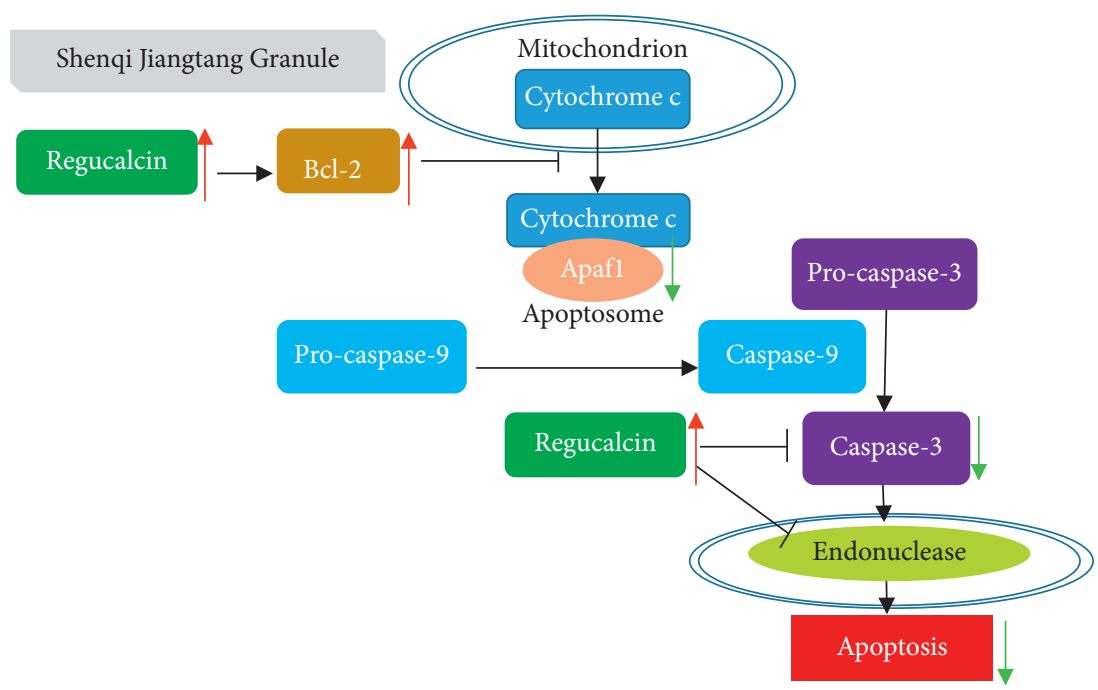

FIGURE 9: Mechanism of SJG inhibition apoptosis on the kidney in diabetic rats. SJG activates regucalcin and Bcl-2. Bcl-2 inhibits the release of cytochrome $\mathrm{c}$ and the formation of the apoptosome with Apaf1, which leads to the inhibition of caspase-9 and subsequent caspase-3. Apaf1: apoptotic peptidase activating factor 1.

(Rgn) and Bcl-2 decreased, while apoptotic peptidase activating factor 1 (Apaf1) and casp3 increased in the diabetic group, compared with the control group $(P<0.01$, Figure 7$)$. SJG treatment increased Rgn and Bcl-2 expression and inhibited Apaf1 and casp3 level expression $(P<0.01$, Figure 7) compared with the diabetic rats. This result was in agreement with the corresponding data from the array.

3.8. SJG Reduced Cell Apoptosis in Diabetic Rats. Kidneys of diabetic rats demonstrated an increased rate of glomerular and tubular cell apoptosis, as shown by TUNEL assay and caspase-3 immunostaining (Figure 8). SJG treatment reduced the rate of apoptosis in both glomerular and tubular cells in diabetic rats (Figure 8) compared with those of the diabetic rats. SJG also inhibited caspase-3 expression in diabetic rat kidney (Figure 8).

\section{Discussion}

In this study, we found that SJG treatment significantly moderated hyperglycemia in diabetic rats. A meta-analysis revealed that SJG reduced FBG and $2 \mathrm{~h}$ postprandial blood glucose in T2DM patients [16]. In addition, our results found that SJG could reduce serum creatinine, BUN, and 24h urinary albumin and could moderate kidney hypertrophy and renal histology in diabetic rats. Previous studies revealed that SJG reduced urinary $\alpha 1$-microglobulin and serum cystatin in early diabetic nephropathy [17].

Shenqi Jiangtang Granule includes Panax ginseng (Panax ginseng C.A. Mey), Radix astragali (Astragalus penduliflorus Lam.), Radix rehmannia (Rehmannia glutinosa (Gaertn.) DC.), Radix trichosanthis (Trichosanthes kirilowii Maxim), Fructus Schisandrae chinensis (Schisandra chinensis (Turcz.) Baill), Ophiopogonis radix (Ophiopogon japonicus (Thunb.) Ker Gawl), Fructus lycii (Lycium chinense $\mathrm{Mill}$ ), Fructus rubi (Rubus chingii $\mathrm{Hu}$ ), Rhizoma dioscoreae
(Dioscorea oppositifolia L.), Alismatis rhizome (Alisma plantago-aquatica subsp.), and Poris cocos (Smilax glabra Roxb). Astragalosides, which is an active ingredient of Radix Astragali (Astragalus penduliflorus Lam), has a potent antioxidative effect and inhibits high glucose-induced mesangial cell proliferation in vitro $[18,19]$. Radix Astragali dramatically reduces oxidative activity in diabetic rat kidneys [19]. Two major isoflavonoids in Radix Astragali has the ability to inhibit AGE-induced endothelial cell apoptosis [20]. Radix rehmannia extract reduced BUN in STZ-induced DN rats [21].

In the gene ontology analysis of differentially expressed genes in the SJG-treated group compared with those in the diabetic group, the regulation of apoptotic process term was ranked second in the biology processes catalog. Pathway analysis of differentially expressed genes also indicated that apoptosis was one of the most enriched pathways. Moreover, TUNEL results also showed that SJG treatment reduced TUNEL-positive cells in diabetic rat kidney glomerular and tubulointerstitial areas compared with those of the diabetic rats. Apoptosis is a process of natural cell death, and it is essential for the development and normal homeostasis of all animals [22]. In the DN rodent model and in patients, hyperglycemia leads to apoptosis in various types of kidney cells, including tubular epithelial cells [23] and endothelial and interstitial cells [24]. Kidney cell apoptosis contributes to loss of kidney function [25]. Taken together, inhibiting kidney cell apoptosis following SJG treatment might be linked to kidney protective effects in diabetic rats.

In gene interaction analysis of differentially expressed genes in the SJG-treated groups compared with those in the diabetic group, Bcl-2, Casp3, and Apaf1 were centrally located within the whole gene interaction network. SJG treatment increased $\mathrm{Bcl}-2$ expression and reduced Casp3 and Apaf1 expression in the kidney compared with that of the diabetic rats. Immunostaining experiments also proved that Caspase-3 expression was reduced in the SJG-treated 
groups, compared with the diabetic group. Mitochondrial apoptosis is regulated by a large number of proteins that directly or indirectly activate or inhibit the activity of cysteine proteases. Bcl-2 is an antiapoptotic regulator [26]. Bcl2 inhibits the release of cytochrome $\mathrm{c}$ and formation of the apoptosome with Apaf1 [27], which lead to the inhibition of caspase- 9 and subsequently of caspase-3 [28]. Previous research revealed that caspase- 3 increases and $\mathrm{Bcl}-2$ decreases in the kidneys of STZ-induced diabetic rats [29]. In immunohistochemical experiments, APAF-1 positivity was increased in diabetic tubular cells [30]. Radix Astragali is one component of SJG. In proximal renal tubular epithelial cells, Astragalosides IV, which is an active ingredient of Radix Astragali, reduced cleaved-caspase-3 expression and increased Bcl-2 expression [31]. Hence, SJG may inhibit mitochondria-dependent apoptosis to moderate kidney function in diabetic rats.

Moreover, we found that SJG activated Rgn expression in diabetic kidneys. Previous research has shown that regucalcin inhibits kidney proximal tubular epithelial cell apoptosis [32]. First, regucalcin causes elevation of Bcl-2 mRNA expression in rat kidney proximal tubular epithelial NRK52E cells [33]. Second, regucalcin inhibited caspase-3 expression in NRK52E cells [34]. Therefore, SJG may inhibit kidney cell apoptosis by activating regucalcin in diabetic rats.

\section{Conclusions}

In summary, our research found a kidney protective effect of SJG in diabetic rats. The mechanism involved may be related to the inhibition of cell apoptosis in the kidney (Figure 9). Inhibition of kidney cell apoptosis may be a potential strategy to treat DN. More experiments in vitro are needed to perform to validate this mechanism.

\section{Abbreviations}

BUN: Blood urea nitrogen

DN: $\quad$ Diabetic nephropathy

ECM: Extracellular matrix

GEO: Gene expression omnibus

GO: Gene ontology

GFR: Glomerular filtration rate

KEGG: Kyoto encyclopedia of genes and genomes

STRING: Search toll for the retrieval of interacting genes

SJG: $\quad$ Shenqi Jiangtang Granule

STZ: $\quad$ Streptozotocin

TUNEL: TdT-mediated dUTP nick-end labeling.

\section{Data Availability}

The data used to support the findings of this study are available from the corresponding author upon request.

\section{Ethical Approval}

Ethical approval for the study was granted by Peking Union Medical Hospital and conformed to the NIH Animal Care guidelines.

\section{Conflicts of Interest}

All authors declare that there are no financial or nonfinancial conflicts of interest regarding the publication of this paper.

\section{Authors' Contributions}

Xinhua Xiao conceived and designed the experiments; Qian Zhang, Jia Zheng, Tong Wang, and Xiaojing Wang performed the experiments; Miao Yu, Ming Li, and Fan Ping analyzed the data; Xinhua Xiao contributed reagents/materials/analysis tools; and Qian Zhang wrote the paper.

\section{Acknowledgments}

This work was supported by the grants from National Key R\&D Program of China (2017YFC1309603), National Key Research and Development Program of China (2016YFA0101002), Medical Epigenetics Research Center, Chinese Academy of Medical Sciences (2017PT31036 and 2018PT31021), the Nonprofit Central Research Institute Fund of Chinese Academy of Medical Sciences (nos. 2017PT32020 and 2018PT32001), National Natural Science Foundation of China (nos. 81170736, 81570715, 81870579, and 81870545), National Natural Science Foundation for Young Scholars of China (no. 81300649), China Scholarship Council Foundation (201308110443), PUMC Youth Fund (33320140022) and Fundamental Research Funds for the Central Universities, and Scientific Activities Foundation for Selected Returned Overseas Professionals of Human Resources and Social Security Ministry. The authors are very grateful to Beijing Compass Biotechnology Company for excellent technical assistance with the microarray experiments.

\section{References}

[1] International Diabetes Federation, IDF Diabetes Atlas, International Diabetes Federation, Brussels, Belgium, 2017.

[2] M. J. Postma and D. de Zeeuw, "The economic benefits of preventing end-stage renal disease in patients with type 2 diabetes mellitus," Nephrology Dialysis Transplantation, vol. 24, no. 10, pp. 2975-2983, 2009.

[3] A. J. Collins, R. N. Foley, B. Chavers et al., "US renal data system 2011 annual data report," American Journal of Kidney Diseases, vol. 59, no. 1, p. A7, 2012.

[4] J. A. Jefferson, S. J. Shankland, and R. H. Pichler, "Proteinuria in diabetic kidney disease: a mechanistic viewpoint," Kidney International, vol. 74, no. 1, pp. 22-36, 2008.

[5] J. W. Baynes, "Role of oxidative stress in development of complications in diabetes," Diabetes, vol. 40, no. 4, pp. 405412, 1991.

[6] M. M. Mariappan, "Signaling mechanisms in the regulation of renal matrix metabolism in diabetes," Experimental Diabetes Research, vol. 2012, Article ID 749812, 10 pages, 2012.

[7] F. A. Hakim and A. Pflueger, "Role of oxidative stress in diabetic kidney disease," Medical Science Monitor, vol. 16, no. 2, pp. RA37-RA48, 2010.

[8] D. Koya, M. R. Jirousek, Y. W. Lin, H. Ishii, K. Kuboki, and G. L. King, "Characterization of protein kinase $\mathrm{C}$ beta isoform activation on the gene expression of transforming growth factor-beta, extracellular matrix components, and prostanoids 
in the glomeruli of diabetic rats," Journal of Clinical Investigation, vol. 100, no. 1, pp. 115-126, 1997.

[9] M. Brownlee, A. Cerami, and H. Vlassara, "Advanced glycosylation end products in tissue and the biochemical basis of diabetic complications," New England Journal of Medicine, vol. 318, no. 20, pp. 1315-1321, 1988.

[10] H. C. Gu, "Clinical observation of Shenqi Jiangtang Keli in treatment of 235 cases of type 2 diabetes," Shandong Yi Yao, vol. 48, p. 116, 2008.

[11] T. L. Y. Wang, "A meta analysis of Shenqi Jiangtang granule treatment on diabetic nephropathy," World Science and Technology, vol. 17, no. 12, pp. 2608-2613, 2015.

[12] Y. Y. X. Huang, "The effect of Shenqi Jiangtang Granule on kidney HIF1-a expression in diabetic rats," Chinese Journal of Integrated Traditional and Western Nephrology, vol. 12, no. 1, pp. 61-62, 2011.

[13] H. Zhang, X. Zhang, H. Jiang, C. Xu, S. Tong, and J. Yan, "Screening and identification of $\alpha$-glucosidase inhibitors from Shenqi Jiangtang Granule by ultrafiltration liquid chromatography and mass spectrometry," Journal of Separation Science, vol. 41, no. 3, pp. 797-805, 2018.

[14] A. M. E. Nahas, S. N. Zoob, D. J. Evans, and A. J. Rees, "Chronic renal failure after nephrotoxic nephritis in rats: contributions to progression," Kidney International, vol. 32, no. 2, pp. 173-180, 1987.

[15] G. Piecha, G. Kokeny, K. Nakagawa et al., "Calcimimetic R-568 or calcitriol: equally beneficial on progression of renal damage in subtotally nephrectomized rats," American Journal of Physiology-Renal Physiology, vol. 294, no. 4, pp. F748-F757, 2008.

[16] J. Z. Fang, Y. Shu, T. Bao et al., “Treating type 2 diabetes mellitus by Shenqi Jiangtang Granule therapy: a meta-anasis of reduce blood glucose efficacy comparion," Journal of Jiangxi University of Traditional Chinese Medicine, vol. 26, no. 5, pp. 32-38, 2014.

[17] D. Wang, "Effect of Shenqi Jiangtang Granule on patients with early diabetic nephropathy of urine alpha protein and serum cystatin 1-microspheres," Clinical Journal of Traditional Chinese Medicine, vol. 29, no. 6, pp. 892-894, 2017.

[18] L. Sun, W. Li, W. Li, L. Xiong, G. Li, and R. Ma, “Astragaloside IV prevents damage to human mesangial cells through the inhibition of the NADPH oxidase/ROS/Akt/NF- $\kappa \mathrm{B}$ pathway under high glucose conditions," International Journal of Molecular Medicine, vol. 34, no. 1, pp. 167-176, 2014.

[19] X. Chen, D.-D. Wang, T. Wei, S.-M. He, G.-Y. Zhang, and Q.-L. Wei, "Effects of astragalosides from Radix Astragali on high glucose-induced proliferation and extracellular matrix accumulation in glomerular mesangial cells," Experimental and Therapeutic Medicine, vol. 11, no. 6, pp. 2561-2566, 2016.

[20] D. Tang, B. He, Z.-G. Zheng et al., "Inhibitory effects of two major isoflavonoids in Radix Astragali on high glucose-induced mesangial cells proliferation and AGEs-induced endothelial cells apoptosis," Planta Medica, vol. 77, no. 7, pp. 729-732, 2011.

[21] T. Yokozawa, H. Y. Kim, and N. Yamabe, "Amelioration of diabetic nephropathy by dried rehmanniae radix (di huang) extract," The American Journal of Chinese Medicine, vol. 32, no. 6, pp. 829-839, 2004.

[22] R. A. Schwartzman and J. A. Cidlowski, "Apoptosis: the biochemistry and molecular biology of programmed cell death," Endocrine Reviews, vol. 14, no. 2, pp. 133-151, 1993.

[23] D. A. Allen, S. Harwood, M. Varagunam, M. J. Raftery, and M. M. Yaqoob, "High glucose-induced oxidative stress causes apoptosis in proximal tubular epithelial cells and is mediated by multiple caspases," The FASEB Journal, vol. 17, no. 8, pp. 908-910, 2003.

[24] W. Dröge, "Free radicals in the physiological control of cell function," Physiological Reviews, vol. 82, no. 1, pp. 47-95, 2002.

[25] E. Adeghate, "Molecular and cellular basis of the aetiology and management of diabetic cardiomyopathy: a short review," Molecular and Cellular Biochemistry, vol. 261, no. 1-2, pp. 187-191, 2004.

[26] B. Vogelstein, D. Lane, and A. J. Levine, "Surfing the p53 network," Nature, vol. 408, no. 6810, pp. 307-310, 2000.

[27] H. Zou, W. J. Henzel, X. Liu, A. Lutschg, and X. Wang, "Apaf1 , a human protein homologous to C. elegans CED-4, participates in cytochrome c-dependent activation of caspase-3," Cell, vol. 90, no. 3, pp. 405-413, 1997.

[28] D. A. Allen, M. M. Yaqoob, and S. M. Harwood, "Mechanisms of high glucose-induced apoptosis and its relationship to diabetic complications," The Journal of Nutritional Biochemistry, vol. 16, no. 12, pp. 705-713, 2005.

[29] N. M. Al-Rasheed, N. M. Al-Rasheed, Y. A. Bassiouni et al., "Simvastatin ameliorates diabetic nephropathy by attenuating oxidative stress and apoptosis in a rat model of streptozotocin-induced type 1 diabetes," Biomedicine \& Pharmacotherapy, vol. 105, pp. 290-298, 2018.

[30] E. Bălăşescu, M. Cioplea, A. Brînzea, R. Nedelcu, S. Zurac, and D. A. Ion, "Immunohistochemical aspects of cell death in diabetic nephropathy," Romanian Journal of Internal Medicine, vol. 54, no. 1, pp. 54-62, 2016.

[31] Q. Chen, Y. Su, Y. Ju, K. Ma, W. Li, and W. Li, “Astragalosides IV protected the renal tubular epithelial cells from free fatty acids-induced injury by reducing oxidative stress and apoptosis," Biomedicine \& Pharmacotherapy, vol. 108, pp. 679686, 2018.

[32] M. Yamaguchi, "The anti-apoptotic effect of regucalcin is mediated through multisignaling pathways," Apoptosis, vol. 18, no. 10, pp. 1145-1153, 2013.

[33] F. J. Dieguez-Acuna, W. W. Polk, M. E. Ellis, P. L. Simmonds, J. V. Kushleika, and J. S. Woods, "Nuclear factor B activity determines the sensitivity of kidney epithelial cells to apoptosis: implications for mercury-induced renal failure," Toxicological Sciences, vol. 82, no. 1, pp. 114-123, 2004.

[34] T. Nakagawa and M. Yamaguchi, "Overexpression of regucalcin suppresses apoptotic cell death in cloned normal rat kidney proximal tubular epithelial NRK52E cells: change in apoptosis-related gene expression," Journal of Cellular Biochemistry, vol. 96, no. 6, pp. 1274-1285, 2005. 


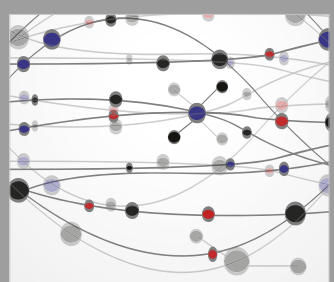

The Scientific World Journal
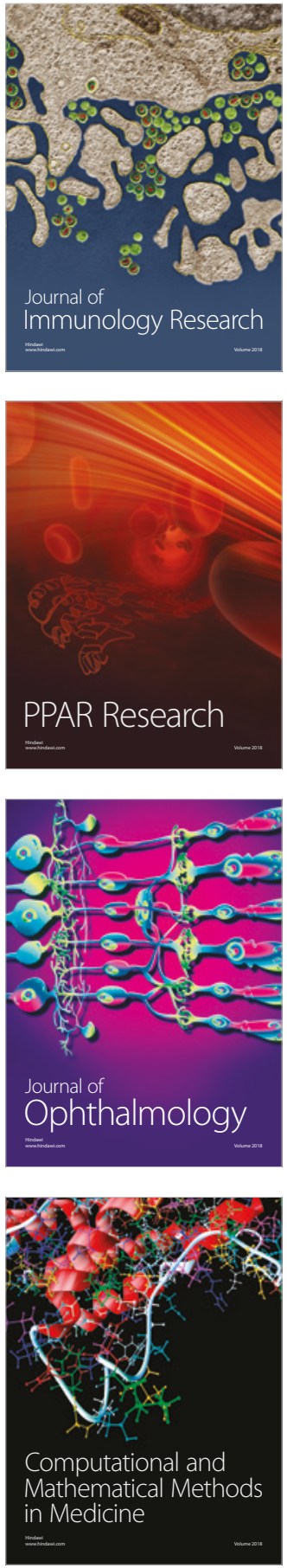

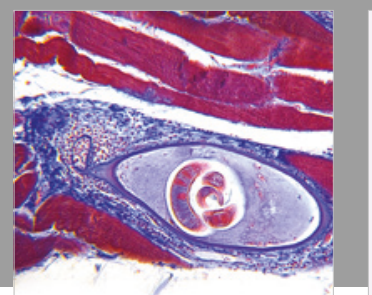

Gastroenterology Research and Practice

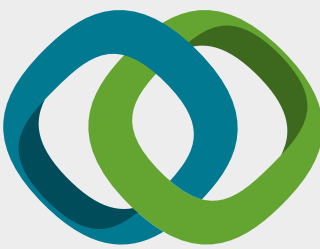

\section{Hindawi}

Submit your manuscripts at

www.hindawi.com
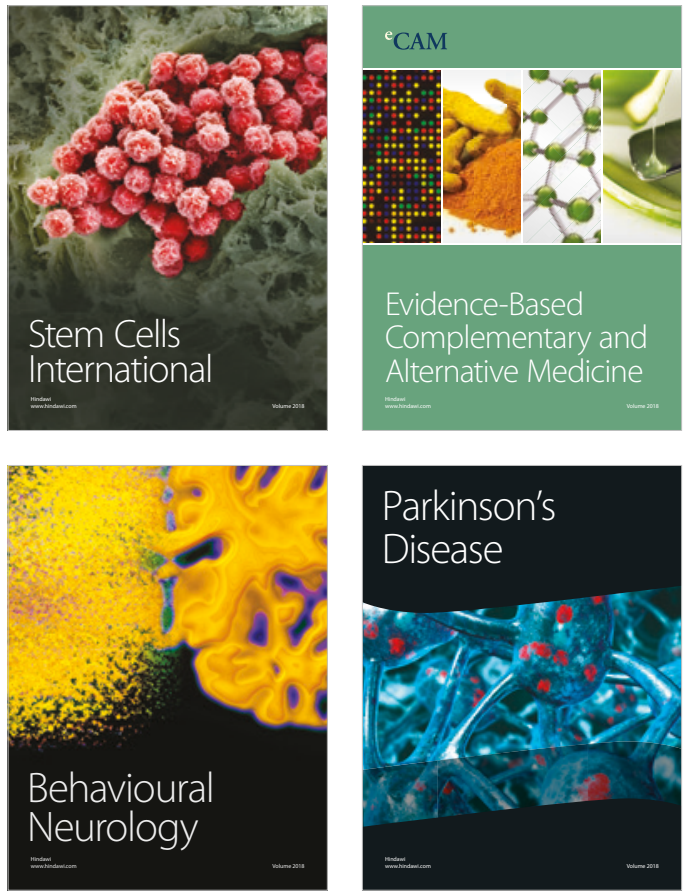

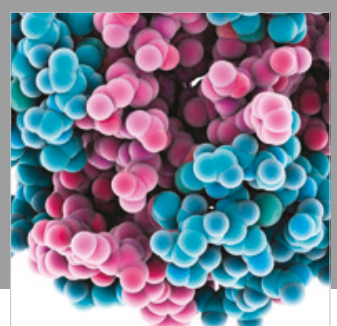

ournal of

Diabetes Research

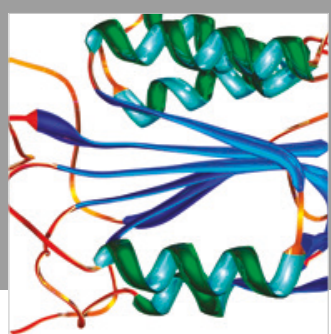

Disease Markers
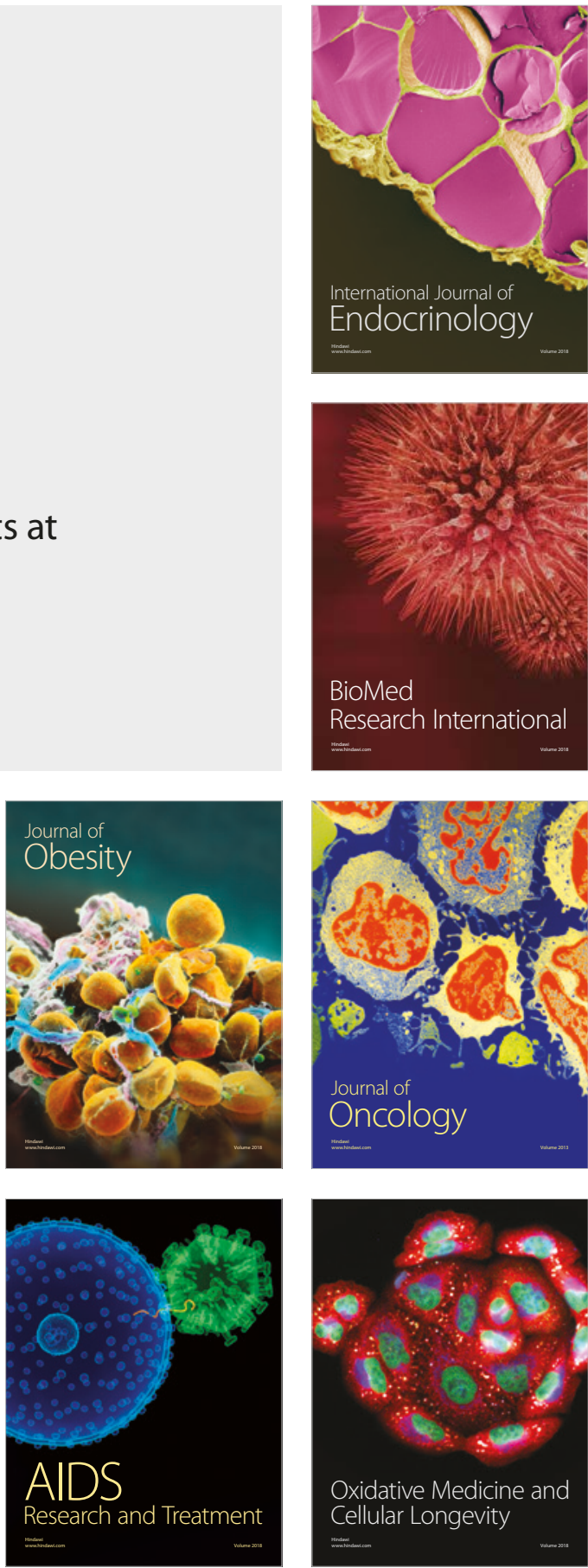